\title{
Comment on "Relativistic Effects of Light in Moving Media with Extremely Low Group Velocity"
}

In 11,2] Leonhardt and Piwnicki have presented an interesting analysis of how to use a flowing dielectric fluid to generate a so-called "optical black hole". Unfortunately there is subtle misinterpretation in the analysis regarding these "optical black holes". While it is clear that "optical black holes" can certainly exist as theoretical constructs, and while the experimental prospects for actually building them in the laboratory are excellent, the particular model geometries of [1, 2] are in fact not black holes at all.

Leonhardt and Piwnicki consider a vortex geometry, where the dielectric fluid is swirling in a purely azimuthal direction around a straight linear core - there is no radial motion into the core in their models, and this is enough to prevent the formation of trapped surfaces and event horizons. This observation depends only on the fact that the effective metric can globally be cast in the ADM-like form

$$
\left[g_{\mathrm{eff}}\right]_{\mu \nu}=\left(\begin{array}{cc}
-\left[c_{\mathrm{eff}}^{2}-g_{a b} v_{\mathrm{eff}}^{a} v_{\mathrm{eff}}^{b}\right] & {\left[v_{\mathrm{eff}}\right]_{i}} \\
{\left[v_{\mathrm{eff}}\right]_{j}} & {\left[g_{\mathrm{eff}}\right]_{i j}}
\end{array}\right) .
$$

In the acoustic geometry of [3, 4, the constant-time 3space metric $\left[g_{\mathrm{eff}}\right]_{i j}$ is particularly simple (it's the identity matrix), the effective velocity $\left[v_{\text {eff }}\right]_{i}$ equals the fluid velocity, and $c_{\mathrm{eff}}$ is just the local speed of sound. In the non-relativistic limit of the non-dispersive movingmedium optical geometry of [2], the effective velocity $\left[v_{\text {eff }}\right]_{i}$ equals the fluid velocity adjusted by the Fresnel drag correction, while $c_{\text {eff }} \rightarrow c / n$ is just the local speed of light, and the 3-metric acquires $O\left(v^{2}\right)$ corrections. In the extreme-dispersion model of [1] the optical geometry is more complicated but the effective velocity is still proportional to the fluid velocity. These technical complications do not affect the key issue: An ergo-region certainly forms once the norm of this effective velocity exceeds the local effective propagation speed $\left(\left\|v_{\text {eff }}\right\|>c_{\text {eff }}\right)$, but provided that $c_{\mathrm{eff}}$ remains positive, a trapped surface will form only if the inward normal component of the effective velocity exceeds the local effective propagation speed [4. Since there is no inward velocity in either of the vortex models [1,2], there is no possibility of forming an event horizon. This result is generic and does not depend on any optical-acoustic analogy.

What Leonhardt and Piwnicki actually do is to demonstrate that the vortex geometries they write down possesses an unstable circular photon orbit, very similar in its qualitative properties to the unstable circular photon orbit that occurs at $r=3 M$ in the Schwarzschild geometry (when the Schwarzschild geometry is written in down in Schwarzschild coordinates.) Photons/lightrays/null geodesics with high angular momentum, higher than some critical value which depends on the details of the fluid velocity profile, either (1) come in from spatial infinity and return to spatial infinity without ever crossing the unstable photon orbit, or (2) emerge from the vortex core, never get past the unstable photon orbit and subsequently fall back to the vortex core. Photons/lightrays/null geodesics with lower than critical angular momentum come in from spatial infinity, cross the unstable photon orbit, and eventually crash into the vortex core. If their angular momentum is just fractionally less than critical they may appear to "hover" near the unstable photon orbit. However, when Leonhardt and Piwnicki use the phrase "Schwarzschild radius" it is the radius of this unstable circular photon orbit they are referring to, and their usage of the phrases "Schwarzschild radius" and "event horizon" has nothing to do with the sense in which they are defined in general relativity.

Despite this technical issue, which causes problems for the two particular toy models they discussed [1,2], it is clear that the basic idea is fine - it certainly is possible to form "optical black holes" but only by adding an inward radial component to the vortex flow. Alternatively, you could think of a nozzle that accelerates a low-compressibility dielectric fluid to (effectively) superluminal velocities. Any region of superluminal effective velocity $v_{\text {eff }}$ will be an ergo-region, and any surface for which the inward normal component of this effective velocity is superluminal will be a trapped surface [4].

If successful, this technique will be able to probe aspects of semiclassical quantum gravity, such as the existence of Hawking radiation. Because the effective metric is not constrained by the Einstein equations you only probe kinematic aspects of how quantum fields react to being placed on a curved background geometry, but do not probe any dynamical questions of just how quantum matter feeds into the Einstein equations to generate real spacetime curvature [4]. Though "effective metric" techniques are limited in this sense, they are still a tremendous advance over the current state of affairs.

\author{
Matt Visser \\ Washington University in Saint Louis \\ Saint Louis, Missouri 63130-4899 \\ e-mail: visser@kiwi.wustl.edu \\ homepage: http://www.physics.wustl.edu/ visser
}

PACS numbers: 42.50.Gy, 04.20.-q

Received 2 Feb 2000; 31 May 2000

[1] U. Leonhardt and P. Piwnicki, Phys. Rev. Lett. 84, 822825 (2000).

[2] U. Leonhardt and P. Piwnicki, Phys. Rev. A 60, 43014312 (1999).

[3] W. Unruh, Phys. Rev. Lett. 46, 1351 (1981); Phys. Rev. D 51, 2827 (1995).

[4] M. Visser, Class. Quantum Grav. 15, 1767 (1998); Phys. Rev. Lett. 80, 3436 (1998); gr-qc/9311028; gr-qc/0003105. 